\title{
A short Survey of Methods for Voltage Instability Detection
}

\author{
Mevludin Glavic, Senior Member, IEEE
}

Thierry Van Cutsem, Fellow, IEEE

\begin{abstract}
This paper shortly surveys existing and proposed methods for voltage instability detection. The emphasis is on methods relying on real-time measurements as well as on longterm voltage instability. Methods are classified according to the required measurement configuration: local vs. wide-area, standard SCADA-type vs. synchronized phasor measurements, etc. In the various categories, some of the features are summarized, and what appears to the authors as advantages or limitations is shortly discussed. An important feature is the ability to anticipate instability. Some tracks for further work on the subject are also outlined.
\end{abstract}

Index Terms - voltage instability, long-term dynamics, widearea monitoring, synchronized phasor measurements, instability detection, state estimation, observability, emergency control

\section{INTRODUCTION}

\section{A. Background}

$\mathbf{P}$ Ower system voltage instability results from the inability of the combined transmission and generation system to deliver the power requested by loads [1], [2]. In many power systems throughout the world, voltage instability is considered as a major threat for secure operation. This paper focuses on long-term voltage instability in which the driving force of instability is load power restoration for instance by Load Tap Changers (LTCs) while the maximum power that the transmission system can provide to loads is reduced by generator reactive power limits, mainly enforced by OverExcitation Limiters (OELs).

In a voltage unstable situation, the voltage drops undergo a dramatic, generally monotonic, decline in the minutes following a disturbance. When this decrease is too pronounced, the system integrity is endangered mainly due to protecting devices that trip generation, transmission, or load equipment. This degradation process may eventually lead to a blackout in the form of a voltage collapse [1], [2].

As a trade-off between security and economy, power systems are usually operated in such a way that they can survive credible incidents (with a reasonable probability of occurrence), while more severe incidents have to be dealt with by System Integrity Protection Schemes (SIPS) [3], [4].

The Phasor Measurement Unit (PMU) technology [5], [6], developed since the end of the 80's, together with advances in computational facilities, networking infrastructure and communications, have opened new perspectives for designing

M. Glavic (glavic@montefiore.ulg.ac.be) is a visiting researcher at the Dept. of Electrical Engineering and Computer Science of the University of Liège, Sart Tilman B37, B-4000 Liège, Belgium.

T. Van Cutsem (t.vancutsem@ulg.ac.be) is a research director of the FRS (Fund for Scientific Research-FNRS) and adjunct professor at the same department. wide-area monitoring systems in general. Voltage stability has been identified as one area where PMU-enhanced functions could prove useful to prevent system blackouts and the associated social and economical losses [5], [7]. For instance, the use of PMU technology for voltage stability monitoring is considered in [7] as one of the critically needed near-future applications.

Nevertheless, adjustments of existing methods or development of new algorithmic solutions are needed in order to fully exploit the advantages offered by those technological advances, and eventually assess the advantages it offers over conventional (but still for some time cheaper) solutions.

The question is thus raised whether technological advances, such as synchronized phasor measurements, could yield enhanced capability of detecting a developing voltage instability with some anticipation. This could serve as early warning signal sent to operators and it could be the heart of an advanced SIPS.

\section{B. The challenge: doing better than simple voltage monitoring}

The curves in Fig 1 show unstable voltages evolutions following a three-phase fault cleared by permanently opening the faulted transmission line. They relate to the test system used in [8]. The initial operating point is insecure with respect to the considered disturbance. The three curves relate to various proportions of induction motor load.

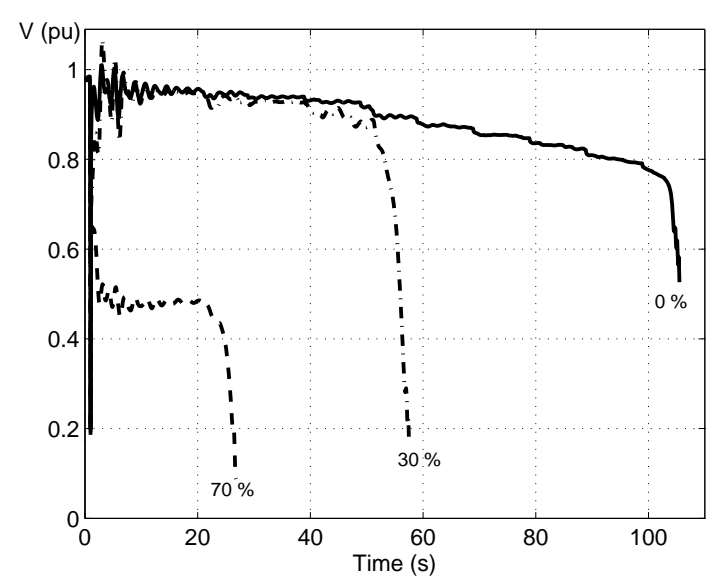

Fig. 1. Unstable evolutions of a transmission voltage when respectively 0 , 30 and $70 \%$ of the loads are represented by equivalent induction motors

The curve with no motor load shows a long-term voltage instability leading to collapse in a bit less than 2 minutes after the fault occurrence. The system evolves under the effect of 
OELs and LTCs. The relatively slow degradation prevailing before the final collapse allows using the shown transmission voltage as triggering signal for emergency controls, such as under-voltage load shedding. The latter acts when the voltage stays below a threshold lying typically between 0.85 and $0.95 \mathrm{pu}$, depending on the system specifics [9], [10]. Load curtailment takes place after a delay of at least 2 to 3 seconds leaving time for voltage to recover after fault clearing (i.e. avoiding to react to normally cleared faults) [11].

In such an instability scenario, the objective of an advanced Voltage Instability Detection (VID) would be to detect the onset of the instability itself, rather than its consequences. Standard SIPS based on fixed voltage thresholds could then be replaced by adaptive schemes, i.e. schemes that would trigger emergency actions at time instants dictated by the instability condition itself. This would also yield an unambiguous identification of instability allowing, for instance, to distinguish an unstable voltage evolution from a low but stable voltage situation.

Coming back to Fig. 1, the curve obtained with $30 \%$ of induction motor load shows a more severe situation where voltage stays at a rather high value, before it drops sharply. This is due to induction motor stalling, caused by the vanishing voltage support from generators switched under field current limit by OELs. In this situation, the voltage decline is so fast that one cannot envisage a SIPS relying on voltage only. Indeed, that protection should react to the voltage decline in a much shorter time than the above mentioned 2 to 3 second delay allowing voltage recovery after fault clearing. One option is to remove this intentional delay upon reception of a signal from neighbouring generators indicating that they are operating above their permanent field current limits [12]. Alternatively, a scheme based on VID could be thought of. However, in this case even more than in the previous one, it is crucial to anticipate the instability before it shows its effects.

Finally, the leftmost curve in Fig. 1 relates to a shortterm voltage instability, where induction motors cannot reaccelerate after the fault has been cleared [1], [2]. To the authors' knowledge there are few publications devoted to the fast detection of short-term voltage instabilities, other than by monitoring low voltage and/or slow voltage recovery [13]. This interesting issue is outside the scope of this paper.

Because monitoring voltage magnitudes is simple and cheap, we believe that the performance of any more advanced VID should be compared to that simple voltage check, in terms of anticipation capabilities. Some publications show indices capable indeed of identifying a voltage unstable situation, but issuing their emergency signal when the system has reached such low voltages that a simple check of the latter would outperform the proposed index.

\section{Scope of the paper}

In this paper we focus on VID methods relying on real-time measurements.

Therefore, we mention only for the sake of completeness the possibility of using faster than real-time simulations in order to diagnose the system post-disturbance evolution, as proposed in [14] and demonstrated in [15] for instance. This is possible with quasi steady-state simulation [2], a fast timedomain simulation method offering a good trade-off between accuracy and computational speed. It can be used to anticipate the near-future response of the system to an equipment outage that has just taken place. This approach requires identifying the outage (which is also the case for some VID methods discussed in this paper) and simulating the latter starting from the pre-disturbance operating point typically given by a state estimator. Of course, the same approach applies to the real-time simulation of an anticipated load increase likely to endanger voltage stability. As a complement, Ref. [14] proposed to couple a wide-area network of PMUs with the real-time model of the system in order to continuously update load model parameters from successive phasor measurements.

In this paper, we do neither discuss the way emergency controls could be initiated from VID, but limit ourselves to outlining the approach proposed in [16] by way of example. This reference suggested to take a snapshot of transmission voltages at the time voltage instability is detected, and use them as threshold voltages in an under-voltage load shedding scheme. The latter, which can be centralized as in [16] or distributed as in [11], curtails blocks of load as long as all transmission voltages are not restored above the thresholds received from VID. This scheme is adaptive in the sense that the voltage thresholds are adjusted to the disturbance, but takes advantage of the robustness and closed-loop operation of the load shedding controller(s).

\section{Classification of methods}

In this paper, we survey a sample of existing or proposed methods for VID using real-time measurements. The survey is certainly non exhaustive, although it should be hopefully representative. Nevertheless, we apologize for the overlooked references and approaches.

The paper is organized with reference to the classification shown in Fig. 2. This classification is based on the underlying real-time measurement configuration, with the "modest" configurations located on the left, and the more demanding ones on the right. The various methods are linked to those configurations by dotted lines. The figure also indicates the sections of the paper in which the methods are summarized or discussed.

\section{VID FROM MEASUREMENTS GATHERED AT ONE LOCATION}

In this section, we focus on VID from measurements gathered at a single location.

\section{A. Approaches based on Thévenin impedance matching}

Voltage instability is linked to the inability of the generation-transmission system to provide the power requested by loads [2]. Therefore, a family of methods aim at detecting maximum load power conditions.

To this purpose, the early work on measurement-based VID and a number of subsequent related contributions referred to 


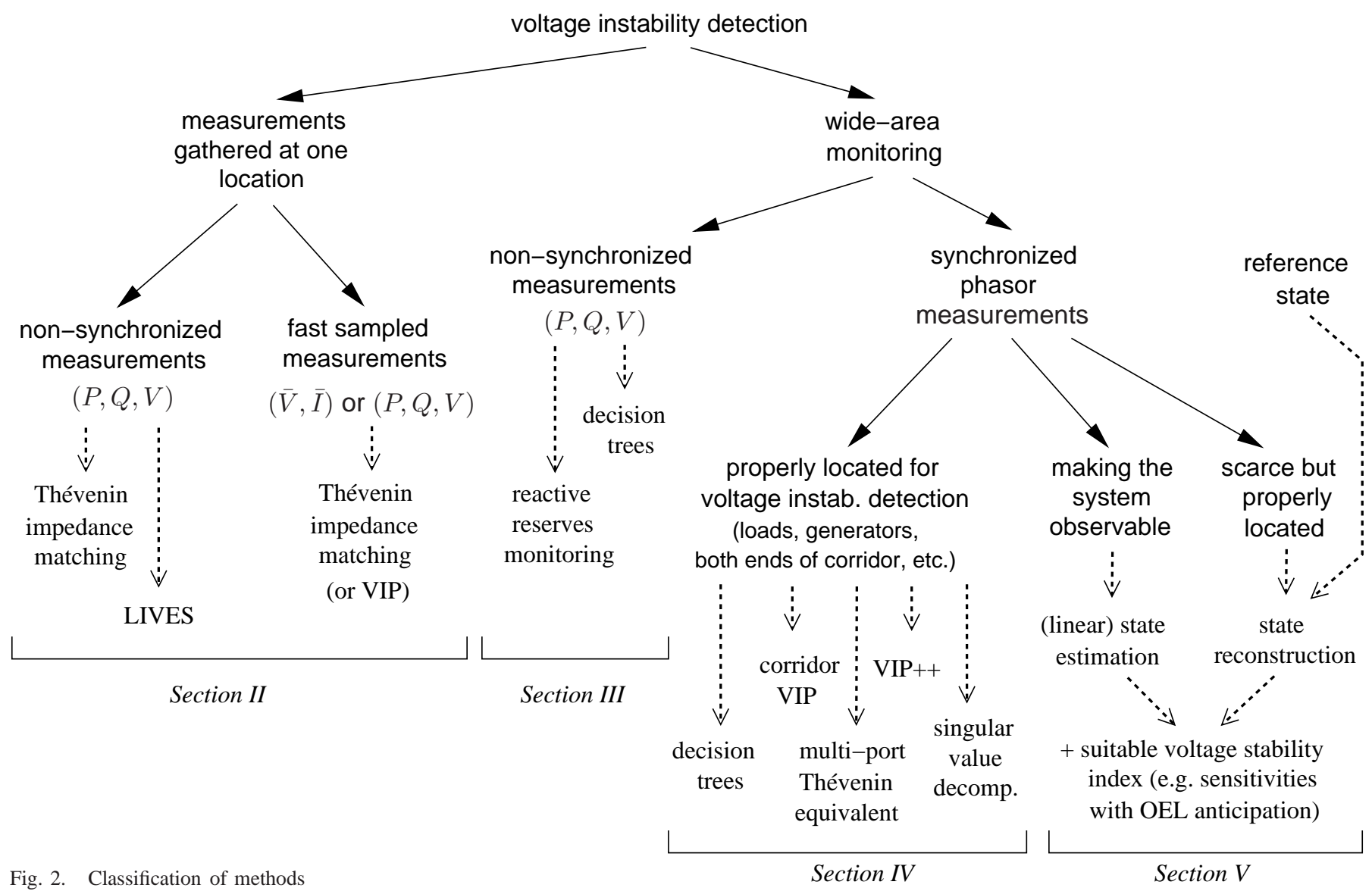

the impedance matching condition [17], [18], [19], which we recall hereafter. The basic assumption is that the whole power system seen from one load can be replaced by a Thévenin equivalent, as shown in Fig. 3. Then, under constant power factor, load power is maximized when the Thévenin and load impedances are equal in magnitude, i.e.

$$
\left|\bar{Z}_{t h}\right|=\left|\bar{Z}_{\ell}\right|
$$

where $\bar{Z}_{t h}$ is shown in Fig. 3, and $\bar{Z}_{\ell}$ is the apparent load impedance, i.e.

$$
\bar{Z}_{\ell}=\frac{\bar{V}}{\bar{I}}
$$

where $\bar{V}$ (resp. $\bar{I}$ ) is the complex voltage across (resp. the complex current in) the load [2]. The maximum load power condition does not involve the assumed power factor but, of course, the active and reactive powers consumed at the maximum depend on that power factor. It is easily shown that $(1,2)$ is equivalent to:

$$
\left|\bar{E}_{t h}-\bar{V}\right|=|\bar{V}|
$$

i.e. the voltage drops in the Thévenin and load impedances are equal in magnitude. A number of practical indices, involving voltage or impedance ratios, load power margins, etc. can be straightforwardly derived from the above condition. A special form of impedance matching condition was introduced in [20], in which a decoupled equivalent network is obtained for every load bus.
Note finally that the Thévenin parameters are expected to change when a generator has its field current limited, since the incremental behaviour of that generator changes dramatically.

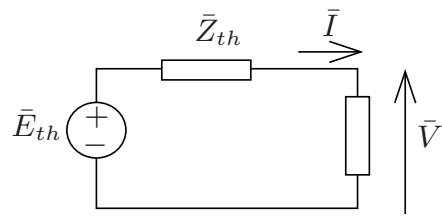

Fig. 3. Thévenin equivalent and load

In the method proposed in [17] the measurements collected at one load bus are used to obtain a Thévenin equivalent of the system seen from that bus, as well as the apparent load impedance $\bar{Z}_{\ell}$. The parameters of the Thévenin equivalent are estimated using a recursive least-square method once two or more measurement sets are available. This method is also known as Voltage Instability Predictor (VIP). The same concept was used in [18] with the proximity to maximum load expressed in terms of power margin.

A simple, single-step calculation procedure, based on two sets of measurements was proposed in [19] to deal with smooth load increases. The method uses an alternative to recursive least-square method in order to compute Thévenin equivalent's parameters. Tellegen theorem and adjoint networks are used to derive a voltage stability index.

Two methods also relying on the Thévenin equivalent were 
presented in [21]. The first method relies on a single Thévenin equivalent, and uses the measurement samples to identify linear relationships between the active and reactive powers of loads; this is used to check a stability limit. The second method identifies two back-to-back Thévenin equivalents at a key transmission bus, for respectively the incoming and the outgoing infeeds.

There is a fundamental issue behind the replacement of a complex, nonlinear, discontinuous power system by a simple Thévenin and the analysis of a multi-dimensional problem from a single load only. This issue was analyzed in [22], where it was shown that the impedance matching condition is necessarily met after meeting the maximum load condition that is reflected into singularity of an appropriate Jacobian matrix.

Another view of the same problem is as follows. At the point tracked by VID, there exists a combination of load powers passing through a maximum. Assume that at this point, we freeze all but one loads and replace the system seen from the last load by a Thévenin equivalent. We expect to find that this load cannot have its power further increased. To this purpose, the Thévenin equivalent should somewhat account for the constant power of the other loads. However, it is unclear how such a nonlinearity could be embedded into a Thévenin equivalent, a notion typical of linear circuits.

There may be practical issues as well. One of them has to do with the use of recursive least squares, which require some care to avoid the so-called "wind-up" effect when there is too little change in system states [23]. On the other hand, fast changes in the Thévenin parameters have to be tracked by selecting a proper value of the forgetting factor involved in recursive least-squares.

Next, the Thévenin impedance has to be estimated from measurements gathered over a time window that should be wide enough for the operating conditions to change, but narrow enough to satisfy the constant Thévenin impedance assumption [24]. This problem was addressed in [25]. By resorting to fast sampled (and higher precision) measurements provided by PMUs, the Thévenin parameters are estimated over a shorter period of time, using an adaptive algorithm. First, the Thévenin e.m.f. is identified based on the observation that this voltage should decrease when the variations in load impedance and estimated Thévenin equivalent reactance are in the same direction, and should increase otherwise. A fivestep algorithm is then proposed to identify the equivalent parameters from an initial value of the reactance and including corrections of the e.m.f..

\section{B. The LIVES method}

A different voltage monitoring method was introduced in [26], further tested in [27] and extended in [28]. The method monitors the distribution voltages controlled by Load Tap Changers (LTCs) on bulk power delivery transformers. Although each LIVES indicator uses the local voltage only, the criterion implicitly accounts for the effect of other taps acting in the system, in so far as it observes the net effect of the various LTCs over a whole cycle of tap operations. An emergency is issued when this net effect is found to move the controlled voltage away from its setpoint value. Thus, LIVES differs from a mere check of the effect of a single tap operation on the corresponding distribution voltage, which would ignore the multi-dimensional aspect of load power restoration by distribution transformers. A moving average is used to filter out measurement noise, as well as to integrate the successive tap effects over the tapping period.

No information is exchanged between the various distribution substations. However, by providing a number of those substations with the indicator, the method shows capability to detect the weak area of the system.

Expectedly, when a monitored LTC hits its limits, the indicator become inactive at the substation of concern [27]. Remedial actions are described in [28].

The handling of significantly different tapping delays, of events impacting distribution voltages in between tap movements and of load power restoration through other than LTCs may deserve further investigations. However, the method already appears attractive as it provides a system-wide indication of instability while relying on local, standard measurements only. Recent progress will be reported in [29].

\section{WIDE-AREA MONITORING USING NON-SYNCHRONIZED MEASUREMENTS}

Recognizing the need to combine information from different locations for reliable VID, methods have been proposed that rely on measurements gathered at two or more locations. We group them under the "wide-area" terminology (see Fig. 2). They are summarized and discussed in this section and the next ones. This section deals in particular with methods using standard, i.e. non-synchronized measurements.

\section{A. Decision Trees}

The use of Decision Tree (DT) methodology for detection of emergency voltage conditions was proposed in [30]. DTs automatically built offline on the basis of learning set and a list of candidate attributes are used in real-time to assess quickly any new operating state, in terms of the values of its test attributes [30]. The latter are readily available from realtime measurements. It was shown in [30] that the DT methodology is able to provide an emergency indicator, automatically generated off-line and efficient to use in real-time.

An extension was proposed in [31], where DTs are introduced to detect voltage instability from the value associated with temporal attributes obtained from direct measurements.

In principle, DTs do not require synchronized measurements; SCADA measurements are enough since time skew should not be critical. However, some recent works proposed taking advantage of PMU data [32], [33], [34], [35] and therefore are shortly presented in the next section.

While using the DT in real-time involves a negligible computational effort, a major practical issue is the ability of setting up a representative learning set. In the context of VID, this set should involve a variety of operating points and disturbances, including both stable and unstable scenarios. This is a key-point but it falls outside the scope of this paper. 


\section{$B$. Reactive reserve monitoring}

Reactive power reserves available on key generators, synchronous generators and static var compensators are sensitive indicators of system stress. One such monitoring scheme, focusing on key system's generators and static var compensators (SVC), is in operation at Bonneville Power Administration [36]. This scheme uses SCADA measurements. Security monitoring relies on normalized effective reactive power reserves computed using an online power flow program.

Another method using var reserves on key generators was introduced in [37]. Although not considered in [37] for VID, it could be used to this purpose through the SCADA system. It was found that reactive reserves of individual sources do not exhibit consistent correlation with the voltage stability margin and the concept of equivalent var reserve, having a good relationship with that margin, was introduced. Furthermore, the method was enhanced with an adaptive learning procedure.

Both [36] and [37] emphasized the need to determine effective reactive power reserves, taking into account that var sources must be close to the affected area to be effective. There is also a need to find a relationship between the reactive power reserves and voltage security. This issue was elaborated in [38], where various reactive power reserve definitions are discussed. The work presented in [39] suggests utilization of statistical multi-linear regression models to transform variations in reactive power reserves into direct information about voltage stability margin.

Reactive reserves from both load and generation perspectives are discussed in [40]. Based on the observation that both reactive reserves are heavily dependent on network, load, generation and system control characteristics, Ref. [40] stresses the need for further work in the definition, computation, valuation and planning of reactive reserves

\section{WIDE-AREA MONITORING USING SYNCHRONIZED}

PHASOR MEASUREMENTS FROM SEVERAL LOCATIONS

This section outlines approaches involving synchronized phasor measurements gathered at several locations.

\section{A. Extensions of Thévenin impedance matching test}

Extensions of the Thévenin impedance matching test using synchronized measurements were proposed in [24], [41], [42].

To avoid the time delay of recursive least-square estimation in VIP, it was proposed in [24], [41] to obtain the Thévenin equivalent from measurements taken at a single time but at both ends of a transmission corridor yielding a wider-area view. After grouping all the lines of the corridor into a virtual link, the parameters of the latter are estimated from measurements taken at both ends. For monitoring purposes, the results of simple calculations are presented in the form of dynamically updated PV curves or active power margins.

An extension of the VIP scheme was described in [42]. In order to improve robustness, this method includes measurements taken at surrounding buses to assist the estimation of the Thévenin equivalent. Moreover, a variant termed VIP++ was introduced, which relies on a prediction of the trajectory to the instability point. This requires estimating the future changes in loads at the PMU-measured buses.

\section{B. Singular Value Decomposition approach}

The method recently proposed in [43] applies singular value decomposition to a measurement matrix. Each column of this matrix includes the measured values provided at a given time by the various PMU devices, and the various columns correspond to successive times (two or three times the number of available PMU measurements). This matrix is updated at each sampling instant with newly acquired measurements. Voltage stability is monitored by computing and tracking the largest singular value of this matrix. This model-free method might be an interesting way to take advantage of a limited number of installed PMUs. Additional theoretical support and validation on realistic systems are needed to demonstrate it as a viable practical solution.

\section{Decision Trees using PMU data}

Several works proposed to use PMU measurements in DTs [32]-[34].

The attributes tested at the various nodes of the DT are PMU data collected at operating points after the initiating disturbance. Different attributes are used in this context: voltage angle differences [32], squares of bus voltages, voltage drops in lines, reactive power flows in lines, currents in lines, generator reactive powers [33]-[35].

Various candidate attributes are combined in [33], [34]. The best performances are obtained using a combination of current magnitudes on branches and fault information. DTs are updated on an hourly basis in order to account for changing system states. The use of multiple optimal DTs and corrective DTs are also proposed and demonstrated in [33], [34].

\section{Multiport Thévenin equivalent}

As stressed in previous sections, generators going under limit significantly impact voltage stability, while multi-load aspects should be also accounted. This leads to preserving generators and loads (at least in the region of interest) instead of lumping all generators and all but one loads into a singleport equivalent. The result is referred to here as a "multiport" Thévenin equivalent.

The idea of collecting information from non-lumped generators is elaborated in [44]. Relying on PMUs covering all generation and load buses, a variant of the impedance matching test (including the non-impedance part of loads into the Thévenin equivalent) together with estimates of generator reactive power reserves is used to identify critical operating conditions.

Under the approximation that a generator can be represented by an e.m.f. of constant magnitude and phase angle behind an equivalent reactance (Ref. [2] discusses the apparent reactance under voltage control and field current limit, respectively), linear circuit analysis can be used. With reference to Fig. 4, using the bus admittance matrix formulation and eliminating the buses with no (significant) load and generator, one easily derives the linear relationship:

$$
\overline{\mathbf{V}}_{\ell}=\mathbf{S} \overline{\mathbf{V}}_{g}-\mathbf{Z} \overline{\mathbf{I}}_{\ell}
$$


where the complex vectors $\overline{\mathbf{V}}_{\ell}, \overline{\mathbf{V}}_{g}$ and $\overline{\mathbf{I}}_{\ell}$ include respectively load bus voltages, generator e.m.f.'s and load currents, while $\mathbf{S}$ is a voltage to voltage sensitivity matrix and $\mathbf{Z}$ an impedance matrix. These matrices change when a generator switches under limit. Equation (4) can be particularized to the $i$-th load bus where the maximum load power condition is tested, treating the other loads as constant power. PMUs are needed to track the values of $\overline{\mathbf{V}}_{g}$. Unless some heuristic simplification is made, $\overline{\mathbf{I}}_{\ell}$ also should be tracked by PMUs.

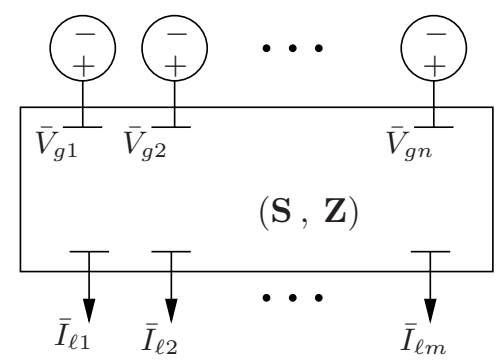

Fig. 4. Multiport Thévenin framework

This formulation can be traced back to [45], where the socalled $L$-index was introduced. At that early time where PMUs were not a reality yet, this index was proposed as a preventive voltage stability margin ${ }^{1}$ but the formulation has been re-used for VID purposes.

A two-bus equivalent is used in [46] where the network behind a load bus is represented by a voltage source in series with a transmission line, preserving power flow and voltage information at the bus of concern. The computation of the equivalent network relies on Eq. (4) and requires PMU measurements at all load and all generation buses.

Ref. [47] proposes a measurement-based voltage stability monitoring relying on the computation of the above $L$ index, and advocating its use in both static and dynamic conditions.

The above quoted references assume rather rich PMU configurations. If such configurations can be afforded, it may be of interest to consider the alternative approaches outlined in the next section.

\section{WIDE-AREA MONITORING RELYING ON ESTIMATED OR RECONSTRUCTED SYSTEM STATE}

The approach discussed in this section corresponds to the rightmost two categories in Fig. 2. It departs from the previous methods in the sense that the measurement configuration is not chosen for VID purposes but rather to estimate the state vector of bus voltages in the whole region subject to voltage instability. Once this state estimate is available, a voltage stability index is computed to detect a developing voltage instability.

${ }^{1}$ Load power margins or secure operation margins are preferred nowadays [2], in particular because the effect of generator limits can be anticipated. The $L$ index yields basically the same information as a load power margin computed under the assumption that no further generator will be limited while the load will increase up to its maximum.

\section{A. State estimation or reconstruction}

In principle the computation of a VID index could rely on bus voltages provided by a standard state estimator processing SCADA measurements. However, SCADA data are collected at a relatively low rate precluding for instance the filtering of measurement noise over a time window. Furthermore, standard (nonlinear) state estimators may encounter convergence problems in degraded system conditions. Last but not least, those data suffer from time skew that could make VID computations unreliable in the presence of transients.

With their higher sampling rate and their negligible time skew, PMUs are likely to improve the quality of presentday state estimation [5]. Although today's power systems are being more and more populated with PMUs, it is not realistic to expect having in a near future full observability by PMUs of a region prone to voltage instability. We can only hope this to become a reality in a farther future. One of the advantages of a PMU-only state estimator lies in the linear relationship between measurements and states, making numerical computations faster and more reliable.

Traditional state estimators, based on SCADA measurements, run every few minutes and provide an average picture of the system state, due to time skew in measurement set. On the other hand, synchronized phasor measurements can potentially track system dynamics between two classical state estimations. However, scarce PMUs do not allow determining the whole system state. Consequently, one of the challenges in effective exploitation of existing or near-future PMU configurations is to somewhat "reconstruct" coherent time-synchronized system states from the available PMU data.

The current work on state reconstruction by the authors is reported in [48]; a summary is given in the Appendix of this paper, for the sake of completeness.

\section{B. VID from estimated or reconstructed system states}

Once an estimate of the bus voltages is available, a good voltage stability index, among those listed in [49], [50] for instance, can be computed.

In Ref. [51] the voltage drop across the Thévenin impedance was approximated by the sum of the absolute values of the complex voltage drops over the branches located on the shortest path from the load bus of concern to a generator under voltage control. The impedance matching criterion is then replaced by a voltage drop matching criterion. Some tuning of the alarm threshold is needed to compensate for the heuristic nature of the method.

Leaving any reference to Thévenin equivalents, an accurate VID can be obtained from singularity conditions of a properly computed Jacobian matrix [2]. In this respect, Ref. [52] proposed a PMU-based technique relying on the Jacobian of the standard power flow equations. Besides the fact that the latter carry some approximation [2], only smooth load increase scenarios were considered.

In the approach of [53] an extended set of equilibrium equations is fitted to the available system state. Then, an efficient sensitivity analysis is performed taking into account the important effects of OELs and LTCs. The sensitivities 
of the total reactive power generation to individual load reactive powers are considered. These sensitivities are used to identify when a combination of load powers passes through a maximum. At this point, the sensitivities change from large positive to large negative values. In theory they should tend to infinity [2] but, in practice, discontinuities and trajectory sampling may prevent them from reaching very high values. Nevertheless the abrupt change in sign provides a clear indication. Another important feature is the anticipation of the OEL activation when forming the Jacobian, which is shown to provide a very good anticipation capability. Extensive tests on snapshots obtained from detailed simulation are reported in [8]. In particular they showed absence of false alarms in marginally stable cases.

\section{CONCLUSION}

In this paper, a sample of approaches for voltage instability detection have been surveyed.

The great majority of works relate to long-term voltage stability. The possibility to use other than voltage measurements when dealing with short-term voltage instability has been comparatively little investigated. This problem, in which short reaction time is a critical issue, might be another field of application of synchronized phasor measurements.

An important feature of voltage instability detection is the capability of anticipatively detecting its onset rather than noticing its consequences. Anticipation can be measured with respect to the time instant where transmission voltages take unacceptably low values, typically the voltage thresholds used in present-day undervoltage load shedding schemes. Of course, this anticipation should not be obtained at the detriment of false alarms, for instance in case of low but stable voltages or in marginally stable scenarios.

It is clear that synchronized phasor measurements have interesting potentialities, but the performance-cost trade-off of any PMU-based scheme should be assessed in comparision with the much simpler check of voltage magnitudes.

In this respect, the approaches which consist of first estimating or reconstructing the system state (vector of bus voltage magnitudes) may be more attractive, in so far as they allow monitoring other aspects of power system security than just voltage stability. Until we reach the time where transmission systems will be covered by PMUs guaranteeing the observability of their states, the problem of estimating or reconstructing the latter from scarce PMU data appears to be very relevant.

The various voltage instability detection methods should be scrutinized on benchmark test systems, assessing the above mentioned trade-offs consistently. Real-time measurements can be simulated from the outputs of detailed time-domain simulation, with addition of measurement noise. This comparative assessment may be worth an IEEE Task Force...

In those tests, voltage instability following an equipment outage should be considered in addition to the more traditional load increase. Indeed, the latter can be monitored through state-of-the-art load power margin calculations. Of main interest is the period that follows the inception of a large disturbance and ends in either a collapse or stable but low voltages.

\section{APPENDIX}

We deal with the problem of reconstructing a system state that satisfies a number of PMU data. We assume that the latter are scarce and, hence, yield an undetermined set of equations.

To solve this indeterminacy, we search for the system state closest to the last estimate provided by a standard state estimator. This minimum distance is defined in the space of load powers. Indeed, even if a disturbance has taken place since the last run of the standard state estimator, the changes in load powers are usually only in the order of a few percents.

Different PMU configurations yield different accuracies of the computed system state. We advocate the placement of PMUs at generator buses for increased accuracy. Indeed, changes in power generations may be large, depending on the reaction to the disturbance of primary frequency and voltage controls. Consequently, measuring the generator voltage and current phasors brings more information about system state.

We thus formulate state reconstruction as the following optimization problem:

$$
\begin{aligned}
\min _{\mathbf{v}_{\mathbf{x}}, \mathbf{v}_{\mathbf{y}}, \mathbf{i}_{\mathbf{x}}, \mathbf{i}_{\mathbf{y}}} & \sum_{i \in \mathcal{N}}\left(P_{i}^{0}-v_{x i} i_{x i}-v_{y i} i_{y i}\right)^{2}+ \\
& +\lambda \sum_{i \in \mathcal{N}}\left(Q_{i}^{0}-v_{y i} i_{x i}+v_{x i} i_{y i}\right)^{2} \\
\text { subject to : } & \mathbf{G}_{\mathbf{v}_{\mathbf{x}}}-\mathbf{B} \mathbf{v}_{\mathbf{y}}-\mathbf{i}_{\mathbf{x}}=\mathbf{0} \\
& \mathbf{B} \mathbf{v}_{\mathbf{x}}+\mathbf{G}_{\mathbf{y}}-\mathbf{i}_{\mathbf{y}}=\mathbf{0} \\
& {\left[\begin{array}{c}
\mathbf{v}_{\mathbf{x}} \\
\mathbf{v}_{\mathbf{y}} \\
\mathbf{i}_{\mathbf{x}} \\
\mathbf{i}_{\mathbf{y}}
\end{array}\right]-\mathbf{b}=\mathbf{0} }
\end{aligned}
$$

where $\mathbf{v}_{\mathbf{x}}, \mathbf{v}_{\mathbf{y}}, \mathbf{i}_{\mathbf{x}}, \mathbf{i}_{\mathbf{y}}$ are vectors of rectangular components of voltages and currents. $\mathbf{G}$ and $\mathbf{B}$ are the real and imaginary parts of the bus admittance matrix. $P_{i}^{0}$ and $Q_{i}^{0}$ are the active and reactive load powers at the $i$-th bus in the reference state. The objective (5a) is the sum of squared deviations between reference and reconstructed load powers. The constraints $(5 b, 5 c)$ are the network equations while $(5 d)$ relates to zero current mismatches (at buses with no load and generator) as well as to the voltage and current phasor measurements provided by the available PMUs. All constraints are thus linear.

\section{REFERENCES}

[1] C. W. Taylor, Power System Voltage Stability, EPRI Power System Engineering Series, McGraw Hill, 1994

[2] T. Van Cutsem, C. Vournas, Voltage Stability of Electric Power Systems, Boston, Kluwer Academic Publishers (now Springer), 1998

[3] D. Karlsson (Convenor), "System Protection schemes in Power Networks," Report of CIGRE Working Group 38.02.19, June 2001.

[4] V. Madani, D. Novosel, S. Horowitz, M. Adamiak, J. Amantegui, D. Karlsson, S. Imai, A. Apostolov, "IEEE PSRC Report on Global Industry Experiences With System Integrity Protection Schemes (SIPS)", IEEE Trans. Power Del., Vol. 20, No. 4, pp. 2143-2155, Oct. 2010

[5] A. G. Phadke, J. S. Thorp, Synchronized Phasor Measurements and Their Applications, Springer, 2008

[6] A. G. Phadke, "Synchronized Phasor Measurements in Power Systems," IEEE Computer Applications in Power, Vol. 6, No. 2, pp. 10-15, Apr. 1993 
[7] D. Novosel, V. Madani, B. Bhragava, K. Vu, J. Cole, "Dawn of the Grid Synhronization: Benefits, Practical Applications, and Deployment Strategies for Wide Area Monitoring, Protection, and Control," IEEE Power and Energy Magazine, pp. 49-60, Jan./Feb. 2008

[8] M. Glavic, T. Van Cutsem, "Wide-Area Detection of Voltage Instability From Synchronized Phasor Measurements. Part II: Simulation Results," IEEE Trans. Power Syst., Vol. 24, No. 3, pp. 1417-1425, Aug. 2009

[9] C. W. Taylor, "Concepts of Undervoltage Load Shedding for Voltage Stability," IEEE Trans. Power Del., Vol. 7, No. 2, pp. 480-488, Apr. 1992

[10] D. Lefebvre, S. Bernard, T. Van Cutsem, "Undervoltage load shedding scheme for the Hydro-Québec system," Proc. IEEE PES General Meeting, Denver (USA), June 2004. Available at http://ieeexplore.ieee.org

[11] B. Otomega, T. Van Cutsem, "Undervoltage Load Shedding Using Distributed Controllers," IEEE Trans. Power Syst., Vol. 22, No. 4, pp. 1898-1907, Nov. 2007

[12] B. Otomega, T. Van Cutsem, "Local vs. wide-area undervoltage load shedding in the presence of induction motor loads," Proc. 2009 IEEE Bucharest Power Tech conference, Bucharest (Romania), June-July 2009. Available at http://ieeexplore.ieee.org

[13] S. M. Halpin, K. A. Harley, R. A. Jones, L. Y. Taylor, "Slope-Permissive Under-Voltage Load Shed Relay for Delayed Voltage Recovery Mitigation," IEEE Trans. Power Syst., Vol. 23, No. 3, pp. 1211-1216, Aug. 2008

[14] C. Rehtanz, J. Bertsch, "Wide Area Measurement and Protection System for Emergency Voltage Stability Control," in Proc. 2002 IEEE Power Eng. Soc. Winter Meeting, New York, NY, pp. 842-847, 2002

[15] T. Van Cutsem, G. Hasse, C. Moors, S. Guillon, and R. Mailhot, "A new training simulator for improved voltage control of the Hydro-Québec system," Proc. IEEE Power System Conf. and Expos. (PSCE), New York, Oct. 2004

[16] M. Glavic, T. Van Cutsem, "Adaptive Wide-Area Closed-Loop Undervoltage Load Shedding Using Synchronized Measurements," Proc. 2010 IEEE Power Eng. Soc. General Meeting, Minneapolis (USA), Jul. 2010

[17] K. Vu, M. M. Begovic, D. Novosel, M. M. Saha, "Use of Local Measurements to Estimate Voltage Stability Margin," IEEE Trans. on Power Systems, Vol. 14, No. 3, pp. 1029-1035, Aug. 1999

[18] D. E. Julian, R. P. Schulz, K. T. Vu, W. H. Quaintance, N. B. Bhatt, D. Novosel, "Quantifying proximity to voltage collapse using the voltage instability predictor (VIP)," Proc. IEEE PES Summer Meeting, Seattle (USA), Jul. 2000, pp. 16-20

[19] I. Smon, G. Verbic, F. Gubina, "Local Voltage-Stability Index Using Tellegen's Theorem," IEEE Trans. on Power Systems, Vol. 21, No. 3, pp. 1267-1275, Aug. 2006

[20] W. Li, T. Chen, W. Xu, "On impedance matching and maximum power transfer," Electric Power Systems Research, Vol. 80, No. 9, pp. 1082-1088, Sep. 2010

[21] M. Parniani, J. H. Chow, L. Vanfretti, B. Bhargava, A. Salazar, "Voltage Stability Analysis of a Multiple-Infeed Load Center Using Phasor Measurement Data," Proc. 2006 IEEE Power System Conference and Exposition, Atlanta, GA, Oct./Nov. 2006

[22] C. D. Vournas, N. G. Sakellaridis, "Tracking Maximum Loadability Conditions in Power Systems," Proc. Bulk Power System Dynamics and Control-VII, Paper 104, Charleston, SC, Aug. 2007

[23] B. Genet, T. Sezi, J-C. Maun, "Comparison of Thevenin's Equivalent Based Methods to Monitor Voltage Stability," Proc. 2008 16th Power System Computation Conference (PSCC), Glasgow, UK, paper 460, Jul. 2008

[24] Zima M., Larsson M., Korba P., Rehtanz C., Andersson G., “Design aspects for wide-area monitoring and control systems," Proceedings of the IEEE, Vol. 93, No. 5, pp. 980-996, 2005

[25] S. Corsi and G. N. Taranto, "A Real-Time Voltage Instability Identification Algorithm Based on Local Phasor Measurements," IEEE Trans. on Power Syst., vol. 23, no. 3, pp. 1271-1279, Aug. 2008

[26] C. D. Vournas, T. van Cutsem, "Local Identification of Voltage Emergency Situations," IEEE Trans. Power Syst., Vol. 23, No. 3, pp. 12391248, Aug. 2008

[27] C. Vournas, N. Sakellaridis, G. Christoforidis, J. Kabouris, T. Van Cutsem, "Investigation of a Local Indicator of Voltage Emergency in the Hellenic Interconnected System", Proc. 16th Power System Computation Conference (PSCC), Glasgow, UK, July 2008.

[28] C. Vournas, C. Lambrou, M. Glavic, T. Van Cutsem, "An integrated autonomous protection system against voltage instability based on Load Tap Changers," Proc. Bulk Power System Dynamics and Control-VIII, Paper 046, Buzios, Brazil, Aug. 2010

[29] C. Lambrou, P. Mandoulidis, C. Vournas, "Local Voltage Instability Monitoring and Protection in the presence of Self-Restoring Loads and
Two-Level LTCs," to be presented at the panel session on Voltage stability monitoring of the 2010 IEEE PES General Meeting, July 2011.

[30] T. Van Cutsem, L. Wehenkel, M. Pavella, B. Heilbronn, M. Goubin, "Decision tree approaches to voltage security assessment," IEE Proc. on Generation, Transmission and Distribution, Vol. 140, No. 3, pp. 189-198, May 1993

[31] P. Geurts, L. Wehenkel, "Temporal machine learning for switching control," Proc. of 4th European Conference of Principles and Practice of Knowledge Discovery in Databases (PKDD), Lyon, France, pp. 401-408, 2000

[32] R. F. Nuqui, "State Estimation and Voltage Security Monitoring Using Synchronized Phasor Measurements," PhD Thesis, Virginia Polytechnic Institute and State University, Blacksburg, Virginia, 2001.

[33] V. Vittal, Decision Tree Based Online Voltage Security Assessment Using PMU measurements, PSERC Report 08-26, Nov. 2008

[34] R. Diao, K. Sun, V. Vittal, R. J. O’Keefe, M. R. Richardson, N. Bhatt, D. Stradford, S. K. Sarawgi, "Decision Tree-Based Online Voltage Security Assessment Using PMU Measurements," IEEE Trans. Power Syst., Vol. 24, No. 2, pp. 832-839, May 2009

[35] M. J. Karki, Methods for Online Voltage Stability Monitoring,. MSc Thesis, Iowa State University, Ames, Iowa, 2009

[36] C. W. Taylor, R. Ramanathan, "BPA Reactive Power Monitoring and Control following the August 10, 1996 Power Failure," Proc. VI Symposium of Specialists in Electgric Operational and Expansion Planning (VI SEPOPE), Salvador, Brazil, May 1998

[37] L. Bao, Z. Huang, W. Xu, "Online Voltage Stability Monitoring Using Var Reserves," IEEE Trans. Power Syst., Vol. 18, No. 4, pp. 1461-1469, Nov. 2003

[38] B. Leonardi, V. Ajjarapu, "Investigation of Various Generator Reactive Power Reserve (GRPR) Definitions for Online Voltage Stability/Security Assessment," Proc. 2008 IEEE PES General Meeting, Tampa (USA), Paper 554, Jul. 2008

[39] B. Leonardi, V. Ajjarapu, "Development of Multilinear Regression Models for Online Voltage Stability Margin Estimation," IEEE Trans. Power Syst., Vol. 26, No. 3, pp. 374-383, Feb. 2011

[40] P. A. Ruiz, P. W. Sauer, "Reactive Power Reserve Issues," Proc. 2006 North American Power Symposium (NAPS), Carbondalle, IL, Paper 121, Sep. 2006

[41] M. Larsson, C. Rehtanz, J. Bertsch, "Real-Time Voltage Stability Assessment of Transmission Corridors," Proc. IFAC Symposium on Power Plants and Power Systems, Seoul, S. Korea, 2003

[42] L. Warland, A. T. Holen, "Estimation of Distance to Voltage Collapse: Testing and Algorithm Based on Local Measurements," Proc. 14th Power System Computation Conference, Paper s38p03, Sevilla (Spain), Jun. 2002

[43] T. Overbye, P. Sauer, C. DeMarco, B. Lesieutre, M. Venkatasubramanian, Using PMU Data to Increase Situational Awareness, PSERC Report 10-16, Sep. 2010

[44] B. Milosevic, M. Begovic, "Voltage Stability Protection and Control using a Wide-Area Network of Phasor Measurements," IEEE Trans. on Power Systems, Vol. 18, No. 1, pp. 121-127, Feb. 2003

[45] P. Kessel, H. Glavitsch, "Estimating the voltage stability of a power system," IEEE Trans. Power Del., Vol. 1, No.3, pp.346-354, July 1986

[46] Y. Gong, N. Schulz, A. Guzman, "Synchrophasor-Based Real-Time Voltage Stability Index," Proc. Power System Conference and Exposition, Atlanta, Oct./Nov. 2006

[47] G. Huang, L. Zhao, "Measurement Based Voltage Stability Monitoring of Power System," PSERC publication 2001, available online: www.pserc.wisc.edu, 2001.

[48] M. Glavic, T. Van Cutsem, "Investigating State Reconstruction from Scarce Synchronized Phasor Measurements," paper accepted for presentation at the IEEE PowerTech conference, Trondheim, June 2011

[49] J. Van Hecke (convenor), N.D. Hatziargyriou, T. Van Cutsem (editors), "Indices predicting voltage collapse including dynamic phenomena," Report of CIGRE working group 38.02.11, CIGRE Publication, 1994, $94 \mathrm{p}$.

[50] C. Canizares (Editor/Coordinator), Voltage Stability Assessment: Concepts, Practices, and Tools, IEEE PES publication, Power System Stability Subcommittee, ISBN 0780378695, 2002.

[51] B. Genet, J-C. Maun, "Voltage Stability Monitoring using WideArea Measurement System," Proc. of IEEE PowerTech Conf., Lausanne (Switzerland), paper 392, Jun. 2007

[52] V. Balamourougan, T. S. Sidhu, M. S. Sachdev, "Technique for online prediction of voltage collapse," IEE Proc. on Generation, Transmission and Distribution, Vol. 151, No. 4, pp. 453-460, Jul. 2004

[53] M. Glavic, T. Van Cutsem, "Wide-Area Detection of Voltage Instability From Synchronized Phasor Measurements. Part I: Principle," IEEE Trans. Power Syst., Vol. 24, No. 3, pp. 1408-1416, Aug. 2009 